Creative arts and digital interventions: A discussion for prevention and recovery from mental health consequences of Adverse Childhood Experiences (ACEs)

Sania Shakoor, Anna Mankee-Williams, Michaela Otis, Kamaldeep Bhui* on behalf of the ATTUNE Collaborative $^{1}$

${ }^{1}$ The ATTUNE Collaborative comprises:

University of Oxford: Prof. Kamaldeep Bhui, Prof. Mina Fazel,

Falmouth University: Prof, Eunice Ma, Ms Anna Mankee Williams, Prof Tanya Krzywinska, Prof. Greg Whelan.

University of Kent: Prof. Nicola Shaughnessy

Leeds University: Prof. Siobhan Hugh-Jones

QMUL: Dr Sania Shakoor, Dr Georgina Hosang, Dr Bridgette Escolme, Dr Michaela Otis

KCL: Prof. Craig Morgan

UCL: Prof. Peter Fonagy, Dr Daisy Fancourt

NSCPCC: Pam Miller

Young People Cornwall: Nick Smith

Cornwall City Council and HEADSTART: Charlotte Hill, Natalie Russell

Centre for Mental Health: Andy Bell

Cornwall Partnership NHS Trust: Prof. Rohit Shankar, Dr Sharon Hudson

*corresponding author 


\title{
Creative arts and digital interventions: A discussion for prevention and recovery from mental health consequences of Adverse Childhood Experiences (ACEs)
}

\begin{abstract}
Adverse childhood experiences (ACEs) are a serious public health concern. They effect mental health across the lifespan, lead to social, emotional and cognitive impairment and reduce life expectancy by up to 20 years. Young people experiencing ACEs are highly vulnerable, and therapeutic service provision has limited capacity and in some places poor access (e.g. isolated rural localities). Research, policy and practice is becoming more cognisant of the needs of people living with the consequences of ACEs. However, individual and social factors (i.e. age, ethnic and gender identity, neurodiversity) are neglected. Arts interventions are recognised as offering significant benefits, especially for culturally diverse groups and young people, and for those with limited literacy or inability to verbally express and articulate their emotional worries. Digital and creative arts interventions offer a range of creative opportunities, involving emotional activation and processing, and all self-determined in terms of the degree of participation in preferred creative activities. So the interventions may be personalised and ensure ACE exposed young people are not re-traumatised and have a positive experience of therapeutic efforts. This paper provides a rapid scoping review of the empirical evidence on creative arts and digital interventions for prevention of ACEs and recovery from the mental health consequences of ACEs.
\end{abstract}




\section{Introduction}

Adverse Childhood Experiences (ACEs) refer to verbal, sexual, or physical abuse; emotional or physical neglect; parental separation or incarceration, problem drug and alcohol use, domestic violence or mental illness in the family; additional experiences that are harmful include bullying, poverty, peer rejection, racism, death and multiple traumatic losses, community violence, food shortages, harsh experiences in care, poor academic performance and living in unsafe environments prior to age 18 years $(1,2)$. Community data from European and Worldwide surveys report high prevalence rates of emotional (29.1\%), physical (22.9\%), and sexual (9.6\%) abuse; as well as physical (16.3\%) and emotional neglect (18.4\%) (3). By the age of eight, 7 in 10 children report at least one ACE and 1 in 10 children report three or more ACEs. ACEs have mental health impacts across the lifespan, and reduce life expectancy by up to 20 years (1). ACEs disrupt neurodevelopment and lead to social, emotional and cognitive impairment and increase risky behaviours, disability and social exclusion (4). Evidence suggests that cumulative exposures to ACEs, rather than individual ACE experiences, have the most significant negative outcomes (2).

Trauma exposed adolescents are more likely to develop complex mental illnesses including major depression, conduct disorder, alcohol dependence, self-harm, suicide attempts, and even posttraumatic stress disorders (PTSD). ACEs can also cause difficulties in education, work, and life-course transitions $(5,6)$. Consequently ACEs have a detrimental economic, social and health cost (7). However, not all young people who experiencing ACES develop these poor outcomes in later life. System-wide or individual supports and resilient characteristics can alleviate the long-term effects of exposure to ACEs (8). Understanding the mechanisms by which ACEs and individual and wider environmental factors impact mental health and influence resilience and recovery are thus important (1).

This rapid scoping review aims to provide a narrative synthesis of the following: 1) What are the risks of mental illness and symptoms amongst young people who have experienced ACEs? 2) How do intersectional influences impact on the magnitude of these risks? 3) How effective are arts-based intervention in supporting young people exposed to ACES? 4) How effective are digital intervention in supporting young people exposed to ACES?

\section{What is the relative risk of mental health illness and its symptomology amongst young people who have experienced ACEs?}

Adverse childhood experiences (ACEs) increase vulnerability for physical, mental and socioeducational adverse outcomes throughout the lifespan $(5,7,9)$. These risks are for male and female survivors $(10,11)$. Meta-analytic evidence suggests a dose-response effect, whereby exposure to four or more ACEs contributes to greater odds of poor health outcomes (12). More specifically data pooled from 37 studies indicated ACEs led to a 2-3 fold increase in the odds of smoking, heavy alcohol use, poor self-rated health, cancer, heart disease, and respiratory disease. ACEs led to a 4-6 fold higher odds of sexual risk and a 7-8 fold greater odds of interpersonal violence (12). Associations have also been reported for multiple illnesses, including major depressive disorder (9) ; bipolar disorder (13); borderline personality disorder (14); anxiety disorders (15) and psychosis (16). 
Efforts to explore the aetiological relationships between ACEs and the wellbeing of survivors have focused on the timing and type of ACEs. Emotional abuse appears to increase the odds of depressive disorders more than physical abuse. In contrast, physical abuse increases the odds of drug use more so than emotional abuse (9). Furthermore, the timing of ACEs differentially impacts on the development of psychopathology. Exposure to ACEs earlier in life (ages 0-5) predicted more symptoms of anxiety and depression in adulthood, in comparison to later onset of ACEs (ages 6-8) which better predicted adult behavioural problems (e.g. substance misuse) (17). In contrast, another study reported exposure to ACEs before the age of 12 contributed to having prominent depressive symptoms, while exposure to ACEs after 12 years of age contributed to a greater risk of having PTSD symptoms (18).

One explanation for the sensitivity of age of onset for ACEs and its consequent impact on mental health comes from neuroscience literature. It is suggested that ACEs influence brain development which in turn contribute to later psychopathology and that the alteration in brain structure, function and connectivity are dependent on the type and timing of exposure (19). Studies have shown that exposure to ACEs is associated with a significant reduction in grey-matter volume in the primary visual cortex amongst those who were survivors of childhood sexual abuse (20), in the primary auditory cortex amongst those who were exposed to parental verbal aggression (21), and in the left anterior and posterior cingulate cortex and bilateral precuneus (regions involved in self- evaluation and selfawareness) amongst those who were exposed to emotional abuse (22). Reduction in the grey-matter volume in these regions contribute to the altered development of sensory systems which process and interpret stress induced by ACEs. This in turn may contribute to the distorted perceptions, cognitions and emotion regulation abilities that are associated with mental illnesses. This suggestion is supported by the observations of reductions in grey-matter volume amongst individuals who have experienced ACEs and those with mental illnesses. In a study of patients with major depressive disorder, a negative correlation between maltreatment severity and grey-matter volume in the bilateral prefrontal cortex was observed (23). Similarly reduction in grey-matter volume in the anterior cingulate cortex has been reported amongst adolescents with PTSD after childhood sexual abuse (24).

ACEs are implicated in the development of emotion dysregulation and biases in emotion recognition (6). In comparison to peers who have not experienced ACEs, individuals who have a history of ACEs report lower levels of emotional understanding (25), higher levels of non-acceptance of emotions (26), distorted perceptions and greater sensitivity to negative facial expressions such as anger and fear (27). These distortions in turn may contribute to the development of mental health disorders rooted in emotional disturbances (i.e. anxiety, depression and PTSD) (28). Similarly ACEs are related to cognitive deficits such as the maladaptive formation of cognitive attributional styles (29), hypersensitivity to threat in ambiguous situations and distortions in social information processing (6). For example being exposed to ACEs repeatedly may contribute to the development of maladaptive cognitive processing, whereby individuals internalise the belief that ACEs are stable, have negative consequences, and are attributable to themselves. This in turn contributes to the development of psychopathology such as depression (30). 


\section{Does the relative risk of mental illness amongst young people who have experienced ACEs, differ across intersectionality factors?}

Although ACEs affect people across ethnicity/race, neurodiversity and socioeconomic divides, the intersecting effects of such factors are key drivers of increased vulnerabilities. The frequency, types and experiences of ACEs are differentially distributed across these groups and associated with disproportionate burdens of trauma amongst some communities (31). The risk of exposure to ACEs is greater amongst those who come from low income or single-parent households, reside in unsafe neighbourhoods and lack access to health care $(8,32)$. Ethnic minority groups, in particular Black and Latino youth are exposed to a greater risk of experiencing ACEs in comparisons to their White peers $(33,34)$. As children and families belonging to ethnic minority groups are more likely to live in lowincome neighbourhoods and experience greater socioeconomic difficulties, the interconnected systems-wide effect of disadvantage may increase vulnerabilities to the effect of ACEs on mental and physical health and dampen recovery (35). Notably, literature demonstrating ethnic disparities in rates of ACEs comes from the United States of America, with a focus on Black and Latino youth $(33,34)$. Studies conducted in the United Kingdom have been underrepresented in ethnic minority populations and consequently not been able to explore the relative risk of ACEs across ethnicity groups $(36,37)$. Albeit limited, there is some evidence to suggest that similar patterns of elevated risks amongst ethnic minorities in the United Kingdom (38). Incidence rates of childhood maltreatment are one and a half times greater amongst those from a Black ethnic minority community in comparison to those from a White ethnic community, and five-fold greater amongst those from the most deprived backgrounds in comparison to those from the least deprived (38).

In addition, neurodiverse individuals with Autism Spectrum Disorder (ASD) and ADHD are more likely to have experienced multiple adverse childhood experiences (ACEs) than neurotypical peers (39-41). Findings extend to differences in clinically relevant cognitive functions such as impairments in general intelligence, executive function, processing speed, memory, perceptual reasoning, and verbal comprehension (42). It is possible that behavioural characteristics associated with these cognitive differences such as impulsiveness, and increased likelihood of interrupting or intruding on others may contribute to vulnerabilities of ACEs such as verbal, physical and peer abuse (43). Higher prevalence of ACEs have also been reported amongst people with disabilities. In a study where disability was measured as limitations in activities due to physical, mental, or emotional problems, or needing specialised equipment such as a cane or a wheelchair due to a health problem, researchers reported that persons with disabilities were more likely to report several ACE categories, particularly childhood sexual abuse(44).

Intersectional factors contribute to the high prevalence of ACEs globally (3). These intersectional factors should be considered in the design of interventions to reduce barriers in service use and therapeutic impacts. More experiential research is needed to move away from the traditional approach of exploring ACEs from a cumulative perspective and move towards developing a more nuanced understanding of ACEs in specific populations, carrying specific vulnerabilities.

\section{How effective are arts-based intervention in supporting young people exposed to ACES?}

Creative arts and arts therapies are emerging as important approaches in the treatment of childhood trauma (45). Many modes of creatives activities such as music, dance, drama and visual arts, artsbased interventions have been utilised to support the well-being of young people and adults (46). 
Evidence suggests that arts-based interventions improve sense of achievement, self-confidence, selfesteem, social skills, conflict resolution, problem solving, relationships and sense of belonging amongst adolescents (46). Equally, these creative activities have been successful in improving mental health and illness recovery amongst adults (47). Studies have found amongst young people who have experienced sexual abuse, music therapy (48) and group art therapy (49) have been effective in reducing depression, anxiety and PTSD symptoms.

It is hypothesised that the participatory and creativity elements of arts-based interventions can contribute to resilience and recovery in a number of ways. For example, art has been found to elicit narrative and facilitate exposure to traumatic cues in a non-threatening and non-invasive manner, this in turn allows for the vocalisation of affective states and reduction in depressive and anxious symptoms $(49,50)$. Arts-based interventions have also been shown to modulate emotional responses to environmental cues and affect mood, through influencing emotional expression and regulation. Using creative practices to express and deal with negative emotions has been found to foster selfesteem, positive relationships and skills for overcoming adversity (51). In addition, the group settings that creative arts-based intervention are often engaged in have the additional benefit of facilitating trust and disclosure amongst the young people partaking. This can provide an opportunity for individuals to realise that they are not alone in their experiences, have a sense of belonging and find peer-support. This can be further enhanced through engaging survivors in co-production projects, whereby participation in the act of 'co-producing' an intervention can have a positive effect by building a sense of belonging and ownership, overcoming stigma, and strengthening social relationships (52).

\section{How effective are digital intervention in supporting young people exposed to ACES?}

Young people are often described as 'digital natives'. This aptitude coupled with the ubiquitous nature of digital technologies (i.e. smart phones and wearables) in modern life, support the therapeutic shift to digital health interventions (DHIs) such as apps, and virtual reality environments. These can increase the accessibility and support available to vulnerable and hard to reach individuals. These advantages alongside anonymity, instant feedback and cost-effectiveness, (53) suggest that digital interventions have the potential to be more helpful and overcome barriers to service use. Furthermore, stigma, fears about confidentiality, shame, financial costs (54) all undermine help seeking; digital approaches may also address the limited numbers of skilled clinicians and therapists in rural areas or in countries with less health spend (low and middle income countries); digital interventions can be used to screen and monitor mental health symptoms, and overcome physical barriers that affect attendance and retention at care services (55). Young people prefer Digital Health Interventions (DHIs) which include features such as videos, limited text, personalisation and the ability to connect with others (56). Thus the shift to using DHIs to support young people exposed to trauma has the potential to be revolutionise care experiences.

It is suggested that digital intervention in the form of serious games may have a positive impact for young people internalising symptoms of mental health (57). Augmented and virtual reality adapted games have been used and found to be helpful for health care design and delivery, in physical and mental health conditions (58). Similarly, digital story telling has been implemented as a narrative intervention when working with survivors of trauma (59). By integrating personalised digital images, text, audio narration, and music, digital story telling supports the processing, organising, and 
integrating of traumatic memories. New narratives are created where the trauma is disentangled from the associated negative thoughts, reminders and emotions. This allows the individual to reclaim power and autonomy to overcome the trauma (59). There is some evidence that digital storytelling is a useful tool to engage with young people who have experienced domestic violence (60) and to capture the lived experience of people who use mental health services. However there is a dearth of research in this area and more is needed to determine effectiveness and optimisation and personalisation for implementation.

Although these approaches show promise for providing therapeutic support to ACE exposed young people $(61,62)$, there are some concerns. Firstly, the experiences of virtual realities are dependent on the inclusion of real-world knowledge to support the ability to engage and feel connected with the virtual environment. The accuracy of the representation of real-world factors in the virtual environment can affect the ability to transfer learnt skills back into real world situations (63). Secondly, through digitalising interventions for traumatic experiences, therapeutic mechanisms such as active listening, empathic understanding and exploration of personal stories may be lost. There may still be a need for supervision, guided use of such interventions, and support to transition from immersive therapeutic technologies, back into ordinary life. Thirdly, there are ethical concerns about triggering disturbing experiences without appropriate support by a clinician or therapist. Lastly, it can be challenging to preserve sufficient personalisation options in the up scaling of interventions delivered via digital platforms. Public and patient involvement at the time of development and testing of digital designs for interventions, could help overcome some of these concerns. For example, including elements of supervised individual and community support and incorporating creative elements in the generating digital content, could offer some reassurance and safeguards. For some, personal therapeutic support may be the only mechanism by which recovery is possible, thus identifying for whom digital interventions may work and how is an important research priority.

\section{Conclusions}

There is a growing shift to digital platforms and increased engagement with virtual realities for therapeutic purposes. This is ever more pertinent due to the increased pressures NHS service provisions experience when providing care to young people who have experienced trauma, and especially so in the current climate of COVID-19 and limited capacity in health systems. Currently there is some evidence on the effectiveness of digital interventions for young people's mental illness, but much more is needed. Further research will need to evaluate the implementation, effectiveness and cost-effectiveness of interventions for a range of axis-I disorders (e.g. ADHD, psychosis and eating disorders), addressing specific social contexts, as well as producing an evaluative taxonomy for digital mental health interventions (64). It is important to consider the mechanistic drivers of trauma related mental illness, and recovery processes. Arts-based interventions show promise for recovery from trauma due to the creative and interactive elements, and such processes may be designed into digital interventions. A number of ethical dilemmas need exploration alongside the intervention design and delivery. The approach we propose is to engage young people in the co-design and implementation of research on digital health interventions.

Acknowledgements: This review was carried out as part of the MARCH Mental Health Network funded by the Cross-Disciplinary Mental Health Network Plus initiative supported by UK Research and Innovation [ES/S002588/1]. 


\section{References}

1. Highland N: Adverse Childhood Experiences, Resilience, and Trauma Informed Care. . in The Annual Report of the DIrector of Public Health2018.

2. Bethell CD, Carle A, Hudziak J, Gombojav N, Powers K, Wade R, Braveman P. Methods to assess adverse childhood experiences of children and families: toward approaches to promote child wellbeing in policy and practice. Acad Pediatr. 2017;17:S51-S69.

3. Sethi D, Bellis M, Hughes K, Gilbert R, Mitis F, Galea G: European report on preventing child maltreatment, World Health Organization. Regional Office for Europe; 2013.

4. Herzog JI, Schmahl C. Adverse Childhood Experiences and the Consequences on Neurobiological, Psychosocial, and Somatic Conditions Across the Lifespan. Front Psychiatry. 2018;9:8.

5. Nurius PS, Green S, Logan-Greene P, Borja S. Life course pathways of adverse childhood experiences toward adult psychological well-being: A stress process analysis. Child Abuse Negl. 2015;45:143-153.

6. Jaffee SR. Child maltreatment and risk for psychopathology in childhood and adulthood. Annual review of clinical psychology. 2017;13:525-551.

7. Bellis MA, Hughes $K$, Ford K, Rodriguez GR, Sethi D, Passmore J. Life course health consequences and associated annual costs of adverse childhood experiences across Europe and North America: a systematic review and meta-analysis. The Lancet Public Health. 2019;4:e517-e528.

8. Bethell CD, Newacheck $P$, Hawes E, Halfon N. Adverse childhood experiences: assessing the impact on health and school engagement and the mitigating role of resilience. Health Affairs. 2014;33:21062115.

9. Norman RE, Byambaa M, De R, Butchart A, Scott J, Vos T. The Long-Term Health Consequences of Child Physical Abuse, Emotional Abuse, and Neglect: A Systematic Review and Meta-Analysis. PLOS Medicine. 2012;9:e1001349.

10. Abajobir AA, Kisely S, Maravilla JC, Williams G, Najman JM. Gender differences in the association between childhood sexual abuse and risky sexual behaviours: A systematic review and meta-analysis. Child Abuse Negl. 2017;63:249-260.

11. Gallo EAG, Munhoz TN, de Mola CL, Murray J. Gender differences in the effects of childhood maltreatment on adult depression and anxiety: A systematic review and meta-analysis. Child Abuse Negl. 2018;79:107-114.

12. Hughes K, Bellis MA, Hardcastle KA, Sethi D, Butchart A, Mikton C, Jones L, Dunne MP. The effect of multiple adverse childhood experiences on health: a systematic review and meta-analysis. The Lancet Public Health. 2017;2:e356-e366.

13. Park Y-M, Shekhtman T, Kelsoe JR. Effect of the type and number of adverse childhood experiences and the timing of adverse experiences on clinical outcomes in individuals with bipolar disorder. Brain sciences. 2020;10:254.

14. Geselowitz B, Whalen DJ, Tillman R, Barch DM, Luby JL, Vogel A. Preschool age predictors of adolescent borderline personality symptoms. Journal of the American Academy of Child \& Adolescent Psychiatry. 2020.

15. McLaughlin KA, Kubzansky LD, Dunn EC, Waldinger R, Vaillant G, Koenen KC. Childhood social environment, emotional reactivity to stress, and mood and anxiety disorders across the life course. Depression and anxiety. 2010;27:1087-1094.

16. Varese F, Smeets F, Drukker M, Lieverse R, Lataster T, Viechtbauer W, Read J, van Os J, Bentall RP. Childhood adversities increase the risk of psychosis: a meta-analysis of patient-control, prospectiveand cross-sectional cohort studies. Schizophrenia bulletin. 2012;38:661-671.

17. Kaplow JB, Widom CS. Age of onset of child maltreatment predicts long-term mental health outcomes. Journal of abnormal Psychology. 2007;116:176.

18. Schoedl AF, Costa MCP, Mari JJ, Mello MF, Tyrka AR, Carpenter LL, Price LH. The Clinical Correlates of Reported Childhood Sexual Abuse: An Association Between Age at Trauma Onset and Severity of Depression and PTSD in Adults. Journal of Child Sexual Abuse. 2010;19:156-170.

19. Teicher MH, Samson JA, Anderson CM, Ohashi K. The effects of childhood maltreatment on brain structure, function and connectivity. Nature Reviews Neuroscience. 2016;17:652-666. 
20. Tomoda A, Navalta CP, Polcari A, Sadato N, Teicher MH. Childhood sexual abuse is associated with reduced gray matter volume in visual cortex of young women. Biological psychiatry. 2009;66:642-648. 21. Tomoda A, Sheu Y-S, Rabi K, Suzuki H, Navalta CP, Polcari A, Teicher MH. Exposure to parental verbal abuse is associated with increased gray matter volume in superior temporal gyrus. Neuroimage. 2011;54:S280-S286.

22. Heim CM, Mayberg HS, Mletzko T, Nemeroff CB, Pruessner JC. Decreased cortical representation of genital somatosensory field after childhood sexual abuse. American Journal of Psychiatry. 2013;170:616-623.

23. Yang S, Cheng Y, Mo Y, Bai Y, Shen Z, Liu F, Li N, Jiang L, Chen W, Lu Y. Childhood maltreatment is associated with gray matter volume abnormalities in patients with first-episode depression. Psychiatry Research: Neuroimaging. 2017;268:27-34.

24. Rinne-Albers MA, Pannekoek JN, van Hoof M-J, van Lang ND, Lamers-Winkelman F, Rombouts SA, van der Wee NJ, Vermeiren RR. Anterior cingulate cortex grey matter volume abnormalities in adolescents with PTSD after childhood sexual abuse. European Neuropsychopharmacology. 2017;27:1163-1171.

25. Shipman K, Zeman J, Penza S, Champion K. Emotion management skills in sexually maltreated and nonmaltreated girls: A developmental psychopathology perspective. Development and psychopathology. 2000;12:47-62.

26. Gratz KL, Bornovalova MA, Delany-Brumsey A, Nick B, Lejuez CW. A laboratory-based study of the relationship between childhood abuse and experiential avoidance among inner-city substance users: The role of emotional nonacceptance. Behavior therapy. 2007;38:256-268.

27. Assed MM, Khafif TC, Belizario GO, Fatorelli R, de Ameida Rocca CC, de Pádua Serafim A. Facial emotion recognition in maltreated children: A systematic review. Journal of Child and Family Studies. 2020;29:1493-1509.

28. Cloitre M, Khan C, Mackintosh M-A, Garvert DW, Henn-Haase CM, Falvey EC, Saito J. Emotion regulation mediates the relationship between ACES and physical and mental health. Psychological Trauma: Theory, Research, Practice, and Policy. 2019;11:82.

29. Gibb BE. Childhood maltreatment and negative cognitive styles: A quantitative and qualitative review. Clinical psychology review. 2002;22:223-246.

30. Schierholz A, Krüger A, Barenbrügge J, Ehring T. What mediates the link between childhood maltreatment and depression? The role of emotion dysregulation, attachment, and attributional style. European journal of psychotraumatology. 2016;7:32652.

31. Nurius PS, Logan-Greene P, Green S. Adverse childhood experiences (ACE) within a social disadvantage framework: Distinguishing unique, cumulative, and moderated contributions to adult mental health. Journal of Prevention \& Intervention in the Community. 2012;40:278-290.

32. Berger LM. Income, family structure, and child maltreatment risk. Children and Youth Services Review. 2004;26:725-748.

33. Liu SR, Kia-Keating M, Nylund-Gibson K. Patterns of adversity and pathways to health among White, Black, and Latinx youth. Child Abuse Negl. 2018;86:89-99.

34. Slopen N, Shonkoff JP, Albert MA, Yoshikawa H, Jacobs A, Stoltz R, Williams DR. Racial disparities in child adversity in the US: Interactions with family immigration history and income. Am J Prev Med. 2016;50:47-56.

35. Chung EK, Siegel BS, Garg A, Conroy K, Gross RS, Long DA, Lewis G, Osman CJ, Messito MJ, Wade Jr R. Screening for social determinants of health among children and families living in poverty: a guide for clinicians. Current problems in pediatric and adolescent health care. 2016;46:135-153.

36. Cawson P, Wattam C, Brooker S, Kelly G. Child maltreatment in the United Kingdom: A study of the prevalence of abuse and neglect. London: NSPCC. 2000.

37. Radford L, Corral S, Bradley C, Fisher HL. The prevalence and impact of child maltreatment and other types of victimization in the UK: Findings from a population survey of caregivers, children and young people and young adults. Child Abuse Negl. 2013;37:801-813. 
38. Chandan JS, Gokhale KM, Bradbury-Jones C, Nirantharakumar K, Bandyopadhyay S, Taylor J. Exploration of trends in the incidence and prevalence of childhood maltreatment and domestic abuse recording in UK primary care: a retrospective cohort study using 'the health improvement network'database. BMJ Open. 2020;10:e036949.

39. Dodds RL. An Exploratory Review of the Associations between Adverse Experiences and Autism. Journal of Aggression, Maltreatment \& Trauma. 2020:1-20.

40. Schneider M, VanOrmer J, Zlomke K. Adverse Childhood Experiences and Family Resilience Among Children with Autism Spectrum Disorder and Attention-Deficit/Hyperactivity Disorder. J Dev Behav Pediatr. 2019;40:573-580.

41. Craig SG, Bondi BC, O'Donnell KA, Pepler DJ, Weiss MD. ADHD and Exposure to Maltreatment in Children and Youth: a Systematic Review of the Past 10 Years. Curr Psychiatry Rep. 2020;22:14.

42. Danese A, Moffitt TE, Arseneault L, Bleiberg BA, Dinardo PB, Gandelman SB, Houts R, Ambler A, Fisher HL, Poulton R. The origins of cognitive deficits in victimized children: implications for neuroscientists and clinicians. American journal of psychiatry. 2017;174:349-361.

43. Gul H, Gurkan CK. Child maltreatment and associated parental factors among children with ADHD: A comparative study. Journal of attention disorders. 2018;22:1278-1288.

44. Austin A, Herrick H, Proescholdbell S, Simmons J. Disability and exposure to high levels of adverse childhood experiences: Effect on health and risk behavior. North Carolina Medical Journal. 2016;77:30-36.

45. van Westrhenen N, Fritz E. Creative arts therapy as treatment for child trauma: An overview. The Arts in Psychotherapy. 2014;41:527-534.

46. Zarobe $L$, Bungay $\mathrm{H}$. The role of arts activities in developing resilience and mental wellbeing in children and young people a rapid review of the literature. Perspect Public Health. 2017;137:337-347. 47. Van Lith T. Art therapy in mental health: A systematic review of approaches and practices. The Arts in Psychotherapy. 2016;47:9-22.

48. Robarts J. Music therapy with sexually abused children. Clinical child psychology and psychiatry. 2006;11:249-269.

49. Pretorius G, Pfeifer N. Group art therapy with sexually abused girls. South African Journal of Psychology. 2010;40:63-73.

50. Lev-Wiesel R, Liraz R. Drawings vs. narratives: Drawing as a tool to encourage verbalization in children whose fathers are drug abusers. Clinical child psychology and psychiatry. 2007;12:65-75.

51. Fancourt D, Garnett C, Spiro N, West R, Mullensiefen D. How do artistic creative activities regulate our emotions? Validation of the Emotion Regulation Strategies for Artistic Creative Activities Scale (ERS-ACA). PLoS One. 2019;14:e0211362.

52. Slay J, Stephens L: Co-production in mental health: A literature review. London, New Economics Foundation; 2013.

53. Olff M. Mobile mental health: a challenging research agenda. European journal of psychotraumatology. 2015;6:27882.

54. Gulliver A, Griffiths KM, Christensen H. Perceived barriers and facilitators to mental health helpseeking in young people: a systematic review. BMC psychiatry. 2010;10:113.

55. Aboujaoude E, Gega L, Parish MB, Hilty DM. Editorial: Digital Interventions in Mental Health: Current Status and Future Directions. Front Psychiatry. 2020;11:111-111.

56. Liverpool S, Mota CP, Sales CM, Čuš A, Carletto S, Hancheva C, Sousa S, Cerón SC, Moreno-Peral $P$, Pietrabissa G. Engaging children and young people in digital mental health interventions: systematic review of modes of delivery, facilitators, and barriers. J Med Internet Res. 2020;22:e16317.

57. Poppelaars M, Wols A, Lichtwarck-Aschoff A, Granic I. Explicit mental health messaging promotes serious video game selection in youth with elevated mental health symptoms. Front Psychol. 2018;9:1837.

58. Fitzgerald M, Ratcliffe G. Serious games, gamification, and serious mental illness: a scoping review. Psychiatric Services. 2020;71:170-183. 
59. Anderson KM, Cook JR. Challenges and opportunities of using digital storytelling as a trauma narrative intervention for traumatized children. Advances in Social Work. 2015;16:78-89.

60. Lamb K, Humphreys C, Hegarty K. Research ethics in practice: challenges of using digital technology to embed the voices of children and young people within programs for fathers who use domestic violence. Research Ethics. 2020:1747016120936324.

61. Boendermaker WJ, Veltkamp RC, Peeters M. Training behavioral control in adolescents using a serious game. Games for health journal. 2017;6:351-357.

62. Jones C, Scholes L, Rolfe B, Stieler-Hunt C. A serious-game for child sexual abuse prevention: an evaluation of orbit. Child Abuse Negl. 2020;107:104569.

63. Milgram P, Takemura H, Utsumi A, Kishino F: Augmented reality: a class of displays on the realityvirtuality continuum. in ProcSPIE1995.

64. Hollis C, Falconer CJ, Martin JL, Whittington C, Stockton S, Glazebrook C, Davies EB. Annual Research Review: Digital health interventions for children and young people with mental health problems - a systematic and meta-review. J Child Psychol Psychiatry. 2017;58:474-503. 